\title{
SI00 calcium binding protein B as a biomarker of delirium duration in the intensive care unit - an exploratory analysis
}

This article was published in the following Dove Press journal:

International Journal of General Medicine

29 November 2013

Number of times this article has been viewed

\author{
Babar A Khan ${ }^{1-3}$ \\ Mark O Farber' \\ Noll Campbell ${ }^{2-5}$ \\ Anthony Perkins ${ }^{2,3}$ \\ Nagendra K Prasad 6 \\ Siu L Hui ${ }^{1-3}$ \\ Douglas K Miller ${ }^{1-3}$ \\ Enrique Calvo-Ayala' \\ John D Buckley' \\ Ruxandra lonescu' \\ Anantha Shekhar' \\ E Wesley Ely 7,8 \\ Malaz A Boustani ${ }^{1-3}$ \\ 'Indiana University School of \\ Medicine, ${ }^{2}$ Indiana University Center \\ for Aging Research, ${ }^{3}$ Regenstrief \\ Institute, Inc., ${ }^{4}$ Wishard Health \\ Services, Indianapolis, ${ }^{5}$ Department of \\ Pharmacy Practice, Purdue University \\ College of Pharmacy, West Lafayette, \\ ${ }^{6}$ Indiana University Melvin and Bren \\ Simon Cancer Center, Indianapolis, \\ IN, ${ }^{7}$ Vanderbilt University School \\ of Medicine, ${ }^{8} \mathrm{VA}$ Tennessee Valley \\ Geriatric Research Education Clinical \\ Center (GRECC), Nashville, TN, USA
}

Correspondence: Babar A Khan Indiana University Center for Aging Research, 410 West 10th Street, Suite 2000, Indianapolis, IN 46202, USA Tel +I 3174235633

Fax +I 3174235695

Email bakhan@iupui.edu
Background: Currently, there are no valid and reliable biomarkers to identify delirious patients predisposed to longer delirium duration. We investigated the hypothesis that elevated S100 calcium binding protein B (S100ß) levels will be associated with longer delirium duration in critically ill patients.

Methods: A prospective observational cohort study was performed in the medical, surgical, and progressive intensive care units (ICUs) of a tertiary care, university affiliated, and urban hospital. Sixty-three delirious patients were selected for the analysis, with two samples of S100 $\beta$ collected on days 1 and 8 of enrollment. The main outcome measure was delirium duration. Using the cutoff of $<0.1 \mathrm{ng} / \mathrm{mL}$ and $\geq 0.1 \mathrm{ng} / \mathrm{mL}$ as normal and abnormal levels of $\mathrm{S} 100 \beta$, respectively, on day 1 and day 8 , four exposure groups were created: Group A, normal S100 $\beta$ levels on day 1 and day 8; Group B, normal S100 $\beta$ level on day 1 and abnormal S100 $\beta$ level on day 8; Group C, abnormal S100 $\beta$ level on day 1 and normal on day 8; and Group D, abnormal S100 $\beta$ levels on both day 1 and day 8 .

Results: Patients with abnormal levels of S100 $\beta$ showed a trend towards higher delirium duration $(P=0.076)$; Group B (standard deviation) (7.0 [3.2] days), Group C (5.5 [6.3] days), and Group D (5.3 [6.0] days), compared to patients in Group A (3.5 [5.4] days).

Conclusion: This preliminary investigation identified a potentially novel role for $\mathrm{S} 100 \beta$ as a biomarker for delirium duration in critically ill patients. This finding may have important implications for refining future delirium management strategies in ICU patients.

Keywords: coma, S100ß, blood-brain barrier, astrocytes

\section{Introduction}

Delirium is a neuropsychiatric syndrome characterized by disturbances in consciousness and a reduced ability to focus, sustain, or shift attention; it occurs over a short period of time and tends to fluctuate over the course of the day. ${ }^{1}$ Delirium complicates care of up to $50 \%$ of mechanically ventilated patients in the intensive care unit (ICU) and is associated with prolonged ICU and hospital length of stay. ${ }^{2,3}$ Duration of delirium in the ICU has been identified as an independent predictor of mortality after adjusting for relevant covariates, including age, severity of illness, comorbid conditions, psychoactive medication use, and baseline cognitive and functional status. ${ }^{4}$ At present, there are no valid and reliable biomarkers associated with delirium duration. Serum biomarkers generated through the pathological processes implicated in delirium may fulfill a role as prognosticators of delirium duration, and may help in identifying candidate patients for early aggressive therapeutic interventions. ${ }^{5}$ 
S100 calcium binding protein B (S100ß) is a protein present in high concentrations in astroglial and oligodendroglial cells in the central nervous system (CNS); a release of $\mathrm{S} 100 \beta$ by these cells may represent glial response to inflammation, ischemia, and metabolic stress. ${ }^{6,7}$ The secretion of $S 100 \beta$ could also be seen as a terminal event of the inflammatory pathway that underlies delirium development and propagation..$^{6-8}$ This pathway highlights the relationship between stress-induced cytokines and astrocytes. ${ }^{8}$ A high systemic inflammatory burden, such as cases where patients are critically ill, predisposes patients to extravasation of leukocytes across the blood-brain barrier and into the CNS parenchyma. ${ }^{9-11}$ Astrocytes are a key component of the blood-brain barrier and their interaction with the cerebrovascular endothelium defines the integrity of the barrier. ${ }^{10,11}$ Thus, an interruption of the blood-brain barrier secondary to inflammation may result in a communication between leukocytes and astrocytes, leading to subsequent activation of astrocytes, with release of $\mathrm{S} 100 \beta$ manifesting clinically as delirium. ${ }^{8}$ Normal S100 $\beta$ levels are very low in the serum of healthy subjects; therefore, high levels may indicate disruption of the blood-brain barrier and astrocyte activation. ${ }^{12-14}$

Elevated serum levels of S100 $\beta$ have been shown to be associated with delirium in both medical and surgical patients, ${ }^{15-19}$ but S100ß's relationship with regards to delirium duration has not been studied in an ICU setting. The majority of critically ill patients have a high systemic inflammatory burden, ${ }^{20,21}$ and delirium is prevalent among the critically ill in ICUs. ${ }^{2,22}$ Hence, the presence of S100 $\beta$ in the serum could serve as a biomarker of astrocyte activation and glial dysfunction secondary to inflammation, and it may provide clues that could explicate delirium duration. We designed this exploratory analysis to investigate the role of $\mathrm{S} 100 \beta$ with regards to delirium duration in patients admitted to the ICU. The primary aim of our study was to test the hypothesis that elevated serum S100 $\beta$ levels are associated with longer delirium duration in critically ill patients.

\section{Methods}

The study was approved by the Institutional Review Board of Indiana University Purdue University at Indianapolis (IUPUI). Informed consent was obtained from patients' legally authorized representatives.

\section{Study setting and patient population}

Patients were included in this prospective cohort analysis if they were admitted to the ICU services of Wishard Memorial
Hospital (WMH) and enrolled in the Pharmacological Management of Delirium (PMD) trial from April 2010 to April 2011. PMD is a National Institutes of Health (NIH)-funded randomized clinical trial ${ }^{23}$ testing the effectiveness of a multi-component intervention to reduce delirium duration and severity in the ICU, details of which have been published previously. ${ }^{24} \mathrm{WMH}$ is a 457-bed, university-affiliated, urban public hospital staffed by Indiana University School of Medicine faculty and house-staff; it serves a population of approximately 750,000 Marion County residents in Indianapolis, Indiana.

The following inclusion criteria were used to enroll patients in the study: 1) admitted to the WMH ICUs; 2) age $\geq 18$ years; 3 ) able to speak English; 4) have delirium based on the Confusion Assessment Method for the ICU (CAM-ICU) ${ }^{25}$ assessments; and 5) have two blood samples collected 1 week apart, first on day 1 and second on day 8 of enrollment in the PMD trial. Patients were excluded if they met any of the following criteria: 1) were not English speaking; 2) were admitted with alcohol intoxication; 3 ) were incarcerated; 4) had a prior history of severe mental illness; 5) were pregnant or nursing; 6) had seizures; 7) had an ischemic or hemorrhagic stroke; or 8) had traumatic brain injury.

\section{Procedures and data collection}

\section{Outcome measures}

The CAM-ICU was used to detect delirium throughout the hospital stay. ${ }^{25}$ Prior to applying the CAM-ICU, the Richmond Agitation Sedation Scale (RASS) ${ }^{26}$ was used to assess patients' eligibility for delirium assessment. Patients were considered delirious if their RASS score was -3 (responsive to verbal stimuli) or greater and if they had a positive CAMICU result: the latter achieved by showing signs of acute change in mental status or fluctuating course, displaying features of inattention, and either disorganized thinking or altered level of consciousness. Legally authorized representatives of eligible delirious patients were approached for enrollment in the PMD trial. As delirium is a fluctuating disorder, our research assistants conducted two delirium assessments each day at different time points to maximize delirium identification. The duration of delirium for each patient was defined as the total number of days the patient was CAM+ on either the morning or evening assessment, throughout the entire hospital stay following enrollment in the study. Coma was defined as a RASS score of -4 (responsive to physical but not to verbal stimulus) or -5 (unresponsive to verbal and physical stimulus) ${ }^{26}$ Delirium resolution was defined as absence of delirium and coma for 2 consecutive 
days (48 hours); persistent delirium was defined as delirium present upon discharge from the hospital.

\section{Biomarker measures}

Two venous blood samples were collected on days 1 and 8 of enrollment under similar conditions; samples were collected between 9 am and $11 \mathrm{am}$, and kept on ice. Serum was obtained by centrifugation for 15 minutes at $4^{\circ} \mathrm{C}$ and aliquots were stored at $-80^{\circ} \mathrm{C}$. S $100 \beta$ levels were measured in duplicate using a prevalidated commercially available assay kit (DiaSorin S.p.A., Saluggia, Italy). This kit employs a two-site one-step enzyme linked immunosorbent assay and includes two positive controls of known concentration (one with $<1.0 \mathrm{ng} / \mathrm{mL}$ and the other with $>2.0 \mathrm{ng} / \mathrm{mL}$ of pure $\mathrm{S} 100 \beta$ antigen), as well as a negative control in every run. Normal values for $\mathrm{S} 100 \beta$ were established as below $0.10 \mathrm{ng} / \mathrm{mL}$ based on a previously published study of 200 healthy volunteers who showed a median plasma concentration of $0.052 \mathrm{ng} / \mathrm{mL}$ (tenth percentile: $0.023 \mathrm{ng} / \mathrm{mL}$; 90th percentile: $0.097 \mathrm{ng} / \mathrm{mL}) .{ }^{27}$ We used the same cutoff points of $<0.1 \mathrm{ng} / \mathrm{mL}$ as normal and $\geq 0.1 \mathrm{ng} / \mathrm{mL}$ as abnormal. For the purpose of study analysis, the study sample was subdivided into four groups based on day 1 and day 8 values of S100ß: Group A, normal S100 $\beta$ levels on day 1 and day 8; Group B, normal S100 $\beta$ level on day 1 and abnormal S100 $\beta$ level on day 8; Group C, abnormal S100 $\beta$ level on day 1 and normal on day 8; and Group D, abnormal S100 $\beta$ levels on both day 1 and day 8 .

\section{Other data collection}

Baseline demographics such as age, sex, and race were collected. Patients' chronic comorbidity status was assessed using the Charlson's comorbidity index. ${ }^{28}$ The validated Informant Questionnaire on Cognitive Decline in the Elderly (IQCODE) ${ }^{29}$ was used to determine patients' previous cognitive function through their surrogates, and the severity of acute illness was assessed using the Acute Physiology and Chronic Health Evaluation (APACHE) II scale. $^{30}$

\section{Statistical analysis}

Variables are presented as mean and standard deviation (SD) for continuous variables and proportions for categorical variables. S100 $\beta$ levels were categorized as normal and abnormal using a cutoff of $0.1 \mathrm{ng} / \mathrm{mL}$. Analysis of variance (for normally distributed data) and Wilcoxon rank sum tests (for skewed data) were used to test for differences in continuous variables among groups defined by S100 $\beta$ levels.
Chi-squared tests were used to test for differences in categorical variables by S100 $\beta$ levels. We used analysis of covariance to investigate the association between S100 $\beta$ level (using the four categories described above) and delirium duration after adjusting for subjects' age, preexisting cognitive impairment status, and severity of acute illness. Since delirium duration is dependent on length of stay and is highly skewed, we used a negative binomial regression model with the natural logarithm of length of stay as an offset variable. We used logistic regression to model the association of S100 $\beta$ level with persistent delirium and in-hospital mortality, and proportional hazards for the association of S100 $\beta$ level with time to delirium resolution. We used a step-down Bonferroni adjustment for multiple comparisons of the individual group when the overall effect was significant. All data analyses were performed using SAS 9.3 software (SAS Institute Inc., Cary, NC, USA).

\section{Results}

A cohort of 63 patients with a total of 126 serum samples (63 samples on day 1 and 63 on day 8 of enrollment) was selected for our analysis. The mean age of the study subjects was 59 ( $\mathrm{SD} \pm 12.6$ ) years with half of the patients being African American. The admission diagnoses of patients included sepsis $(22 \% ; 14 / 63)$, acute respiratory failure $(11 \%$; $7 / 63)$, chronic obstructive pulmonary disease/asthma $(6 \%$; $4 / 63)$, altered mental status $(5 \% ; 3 / 63)$, congestive heart failure/arrhythmia $(5 \% ; 3 / 63)$, hypertension $(5 \%, 3 / 63)$, renal failure $(3 \% ; 2 / 63)$, burns $(3 \% ; 2 / 63)$, malignancy $(3 \% ; 2 / 63)$, esophageal/gastric perforation $(3 \% ; 2 / 63)$, hernia repair $(3 \%$; $2 / 63)$, small bowel surgery $(3 \% ; 2 / 63)$, and others $(27 \%$; 17/63). Table 1 describes the baseline characteristics of the study population as a function of the four groups based on S100 $\beta$ levels on day 1 and day 8 . There were no significant differences among the groups with respect to age, sex, ethnicity, chronic comorbidities, preexisting cognitive impairment, and severity of illness.

Groups with abnormal values of $\mathrm{S} 100 \beta$ saw a higher duration of delirium during the overall hospital stay when adjusted for age, preexisting cognitive impairment, and severity of illness $(P=0.076)$, as shown in Table 2. As the overall $P$-value approached but did not reach significance, a pairwise comparison between groups was not performed. The rates of persistent delirium were also found to be different among the four groups $(P=0.004)$. A pair-wise comparison showed that patients in Group B were more likely to be discharged with delirium (60\%) when compared to Groups A $(0 \%, P=0.015)$ and $\mathrm{C}(0 \%, P=0.036)$, but not with Group D 
Table I Baseline characteristics of patients in the overall cohort and based on their SI00 $\beta$ values on day I and day 8

\begin{tabular}{|c|c|c|c|c|c|c|}
\hline \multirow[t]{3}{*}{ Characteristic } & \multirow{3}{*}{$\begin{array}{l}\text { Overall } \\
n=63\end{array}$} & \multicolumn{5}{|l|}{ SI $00 \beta$ levels } \\
\hline & & \multirow{2}{*}{$\begin{array}{l}\text { Group A } \\
\text { *Normal day I, } \\
\text { normal day } 8 \\
(n=16)\end{array}$} & \multirow{2}{*}{$\begin{array}{l}\text { Group B } \\
\text { Normal day I, } \\
\text { abnormal day } 8 \\
(n=5)\end{array}$} & \multirow{2}{*}{$\begin{array}{l}\text { Group C } \\
\text { \#Abnormal day I, } \\
\text { normal day } 8 \\
(n=I I)\end{array}$} & \multirow{2}{*}{$\begin{array}{l}\text { Group D } \\
\text { Abnormal day I, } \\
\text { abnormal day } 8 \\
(n=3 I)\end{array}$} & \multirow[t]{2}{*}{$P$-value } \\
\hline & & & & & & \\
\hline Age, mean (SD) & $59(12.6)$ & $54.2(15.2)$ & $57.1(5.5)$ & $60.2(13.1)$ & $61.5(11.4)$ & 0.293 \\
\hline Female, \% (n) & $61.9(39)$ & $56.2(9)$ & $60.0(3)$ & $72.7(8)$ & $61.3(19)$ & 0.855 \\
\hline African American, \% (n) & $47.6(30)$ & $43.7(7)$ & $40.0(2)$ & $36.4(4)$ & $54.8(17)$ & 0.701 \\
\hline IQCODE, mean (SD) & $3.2(0.4)$ & $3.0(0.1)$ & $3.4(0.5)$ & $3.2(0.5)$ & $3.2(0.4)$ & 0.251 \\
\hline $\begin{array}{l}\text { Charlson comorbidity index, } \\
\text { mean (SD) }\end{array}$ & $3.2(2.7)$ & $2.1(2.0)$ & $4.2(2.4)$ & $2.8(2.8)$ & $3.6(2.9)$ & 0.228 \\
\hline APACHE II score, mean (SD) & $16.4(6.9)$ & $15.9(7.9)$ & $17.8(2.4)$ & $18.1(10.1)$ & $15.9(5.6)$ & 0.791 \\
\hline Sepsis, \% (n) & $22.2(14)$ & $18.8(3)$ & $40.0(2)$ & $18.2(2)$ & $22.6(7)$ & 0.769 \\
\hline $\begin{array}{l}\text { Duration of mechanical } \\
\text { ventilation, days }(S D)\end{array}$ & $8.4(9.8)$ & $6.7(6.9)$ & $8.0(6.8)$ & $5.9(8.3)$ & $10.3(11.8)$ & 0.443 \\
\hline Service & & & & & & 0.867 \\
\hline MICU, \% & 71.4 & 75.0 & 80.0 & 63.6 & 71.0 & \\
\hline SICU, \% & 17.5 & 18.8 & 0.0 & 27.3 & 16.1 & \\
\hline PICU, \% & 11.1 & 6.2 & 20.0 & 9.1 & 12.9 & \\
\hline
\end{tabular}

Notes: *Normal SI00 $\beta$ levels were defined as $<0.1 \mathrm{ng} / \mathrm{mL}$; \#abnormal SI00 $\beta$ levels defined as $\geq 0.1 \mathrm{ng} / \mathrm{mL}$.

Abbreviations: n, number; SD, standard deviation; IQCODE, Informant Questionnaire on Cognitive Decline in the Elderly; APACHE, Acute Physiology and Chronic Health Evaluation; MICU, medical intensive care unit; SICU, surgical intensive care unit; PICU, progressive "step-down" intensive care unit.

(12.9\%, $P=0.558)$. Patients in Groups A and $\mathrm{C}$ with normal S100ß values on day 8 had $100 \%$ delirium resolution compared to Groups B and D with abnormal day 8 S100ß levels (60\% and $83.9 \%$, respectively); however, this was not statistically significant $(P=0.183)$. Hospital and ICU lengths of stay, as well as in-hospital mortality, did not differ significantly among groups, although the point estimates of all outcomes appeared to be better in the two groups with normal S100 $\beta$ levels on day 8 .

\section{Discussion}

Our preliminary results are suggestive of a relationship between elevated S100 $\beta$ levels and longer delirium duration in critically ill patients, after adjustment for

Table 2 Association between SIO0 $\beta$ day I and day 8 levels with delirium duration, resolution, and persistence, length of stay, and mortality

\begin{tabular}{|c|c|c|c|c|c|c|}
\hline \multirow[t]{3}{*}{ Outcomes } & \multicolumn{4}{|l|}{ SI00ß levels $\#$} & \multirow[t]{3}{*}{$P$-value } & \multirow{3}{*}{$\begin{array}{l}\text { Adjusted } \\
\text { P-value* }\end{array}$} \\
\hline & \multirow{2}{*}{$\begin{array}{l}\text { Group A } \\
\text { Normal day I, } \\
\text { normal day } 8 \\
(n=16)\end{array}$} & \multirow{2}{*}{$\begin{array}{l}\text { Group B } \\
\text { Normal day I, } \\
\text { abnormal day } 8 \\
(n=5)\end{array}$} & \multirow{2}{*}{$\begin{array}{l}\text { Group C } \\
\text { Abnormal day I, } \\
\text { normal day } 8 \\
(n=I I)\end{array}$} & \multirow{2}{*}{$\begin{array}{l}\text { Group D } \\
\text { Abnormal day I, } \\
\text { abnormal day } 8 \\
(n=3 I)\end{array}$} & & \\
\hline & & & & & & \\
\hline $\begin{array}{l}\text { Delirium duration (days), } \\
\text { mean (SD) }\end{array}$ & $3.5(5.4)$ & $7.0(3.2)$ & $5.5(6.3)$ & $5.3(6.0)$ & 0.067 & 0.076 \\
\hline $\begin{array}{l}\text { Delirium/coma duration (days), } \\
\text { mean (SD) }\end{array}$ & $5.4(7.5)$ & $10.6(4.4)$ & $8.1(9.1)$ & $9.3(8.6)$ & 0.143 & 0.112 \\
\hline $\begin{array}{l}\text { Coma duration (days), } \\
\text { mean (SD) }\end{array}$ & $2.3(2.8)$ & $3.6(3.6)$ & $3.2(5.1)$ & $4.3(5.4)$ & 0.649 & 0.567 \\
\hline Delirium resolution, ${ }^{a} \%$ (n) & $100.0(16)$ & $60.0(3)$ & $100.0(11)$ & $83.9(26)$ & 0.147 & 0.183 \\
\hline Persistent delirium, ${ }^{b} \%$ (n) & $0.0(0)$ & $60.0(3)$ & $0.0(0)$ & $12.9(4)$ & 0.004 & $* *$ \\
\hline $\begin{array}{l}\text { Hospital length of stay (days), } \\
\text { mean (SD) }\end{array}$ & $26.8(18.1)$ & $31.8(27.1)$ & $22.8(13.3)$ & $34.3(20.9)$ & 0.248 & 0.611 \\
\hline $\begin{array}{l}\text { ICU length of stayc (days), } \\
\text { mean (SD) }\end{array}$ & $15.9(\mid 1.2)$ & $12.4(7.4)$ & I3.I (9.3) & $19.7(12.4)$ & 0.163 & 0.400 \\
\hline In-hospital mortality, \% (n) & $0.0(0)$ & $20.0(I)$ & $0.0(0)$ & I6. I (5) & 0.168 & $* *$ \\
\hline
\end{tabular}

Notes: "Normal SI00 $\beta$ levels were defined as $<0.1 \mathrm{ng} / \mathrm{mL}$, abnormal levels were defined as $\geq 0.1 \mathrm{ng} / \mathrm{mL}$; *adjusted for age, preexisting cognitive impairment and severity of illness; ${ }^{a}$ delirium resolution was defined as absence of delirium/coma for 48 hours; ${ }^{b}$ persistent delirium was defined as delirium present upon discharge; ‘CU length of stay excluded the stay in the progressive unit; **too few outcomes for modeling.

Abbreviations: $n$, number; SD, standard deviation; ICU, intensive care unit. 
relevant confounders. Patients with abnormal levels of S100 $\beta$ either on day 1 or day 8 (or both) were found to have higher delirium duration compared to patients with normal S100 $\beta$ levels on both days. Prior studies have demonstrated an association between the presence of delirium and elevated S100 $\beta$ levels, ${ }^{15-19}$ our study adds to this literature by exploring the role of $S 100 \beta$ as a marker for delirium duration. As delirium duration is an independent predictor of higher mortality, ${ }^{4}$ identifying patients with a predisposition towards longer delirium duration might be useful in instituting more effective and personalized therapies, with the end goal of decreasing the burden of delirium. Similarly, recognizing patients with a tendency for persistent delirium may help in developing appropriate discharge plans for patients with such tendencies.

Our study findings are helpful in understanding the role activated astrocytes may play in the overall pathophysiology of delirium. Elevated levels of S100 $\beta$ later in the disease course bring out the theoretical possibility that persistent astrocyte activation is somehow associated with longer delirium duration. Coupled with the finding that normal S100 $\beta$ levels on day 8 correlate with delirium resolution, this points towards there being a role for activated astrocytes in sustaining delirium. As patients with underlying CNS processes were not included in the analysis, the elevated S100 $\beta$ levels do not reflect an overt neuronal damage phenomenon, but most likely a state of astrocyte activation, dysfunction, and permeability of the blood-brain barrier; however, extracerebral sources of $\mathrm{S} 100 \beta$ elevation cannot be excluded. ${ }^{31-34}$ As the first blood draw in our study was collected at a time when patients were already delirious, it is difficult to ascertain whether delirium led to astrocyte activation or the astrocyte activity resulted in delirium symptoms. Our study was not designed to answer this question but was instead intended to identify $\mathrm{S} 100 \beta$ as a marker associated with delirium duration. Based on our preliminary findings, it may be worthwhile to explore $\mathrm{S} 100 \beta$ further in future studies as a biomarker of delirium duration.

Prior work by Nguyen et al showed that elevated S100 $\beta$ levels are associated with encephalopathy in critically ill septic patients. ${ }^{35}$ The authors classified encephalopathy as Type A (agitation, confusion, irritability, convulsions) and Type B (somnolence, stupor, coma). Elevated S100 $\beta$ levels were associated with Type B but not with Type A encephalopathy. As delirium was not assessed with a validated delirium scale, the question remains whether the diagnosis of encephalopathy represented delirium in this patient population. It may be plausible that the classification scheme of Type A and Type B encephalopathy represented hyper- and hypoactive delirium, respectively. The relationship between elevated $\mathrm{S} 100 \beta$ and delirium in the ICU has not been consistent with some data showing no association between this biomarker and delirium occurrence. ${ }^{36}$ Absence of a control group precludes us from commenting on $\mathrm{S} 100 \beta$ and its relation to delirium incidence, but based on our preliminary data, we can hypothesize that fluctuations in S100 $\beta$ levels between normal and abnormal may explicate delirium duration. However, this hypothesis needs future validation in larger cohort studies.

\section{Strengths and limitations}

The major strengths of our study include a diverse patient population (including females and African American patients), two blood draws collected at different time-points of the disease course, adjustment of relevant confounders in the final analysis, and twice-daily use of reliable and validated sedation/delirium assessment tools. In addition, our study fulfills the major requirements of reporting prognostic marker studies, ${ }^{37}$ including adequate blinding in the evaluation of the prognostic marker to the outcome (and vice versa), a prospective design, utilization of standardized tools for outcome assessment (eg, the CAM-ICU for identifying delirium), description of the biomarker assay (with appropriate reference), and inclusion of the whole length of patients' stays in the hospital.

Our study has several limitations. Firstly, a sample size of 63 patients is too small to study all meaningful outcomes and associations. Such a sample size does not have enough power to detect group differences of outcomes with large variances (eg, length of stay), and it precludes the evaluation of differences in various subgroups, such as surgical or medical ICU patients and presence or absence of mechanical ventilation. Secondly, our second blood draw, which was a week after enrollment, also proved to be relevant to delirium duration. Blood drawn earlier, such as on the second or third day of enrollment, may have provided the same prognostic information, albeit at an earlier stage. A prospective study with daily blood samples should be able to answer this question. Given the limited sample size, we were only able to include three covariates in our final analysis. We did not have drug dispensing data, and so the exposure of the cohort to sedatives and analgesics could not be determined; an uneven drug distribution among groups could have confounded the results. There were no CNS S100 $\beta$ measurements to document a correlation with the serum samples. Since we selected patients with two blood draws 
1 week apart, the findings of our study are only applicable to patients with a considerably longer duration of hospital stay. Although CAM-ICU has been shown to be valid and reliable in ICU patients, its sensitivity outside the ICU is low. Finally, concomitant measurement of inflammatory markers such as interleukins and C-reactive protein would have further highlighted the interaction between inflammation and astrocyte activation.

\section{Conclusion}

In conclusion, our pilot study suggests that there may be an association between elevated S100 $\beta$ levels and longer delirium duration and delirium persistence in critically ill ICU patients. Future studies with larger sample sizes and daily blood draws are required to validate these preliminary findings.

\section{Disclosure}

The study was supported by a grant from the National Institute on Aging (R01AG034205) and National Institute of Mental Health (R24MH080827). Dr Khan's work on the project was supported through a Career Development Award from the National Institute on Aging (NIA K23-AG043476). Dr Siu Hui's and Dr Anantha Shekhar's work on the project was supported by the Indiana Clinical and Translational Sciences Institute, funded in part by the National Center for Research Resources and National Center for Advancing Translational Sciences, National Institutes of Health (Grant RR025761). The content is solely the responsibility of the authors and does not necessarily represent the official views of the NIH. The remaining authors do not report any additional conflicts of interest.

\section{References}

1. Inouye SK, van Dyck CH, Alessi CA, Balkin S, Siegal AP, Horwitz RI. Clarifying confusion: the confusion assessment method. A new method for detection of delirium. Ann Intern Med. 1990;113(12):941-948.

2. Khan BA, Guzman O, Campbell NL, et al. Comparison and agreement between the Richmond Agitation-Sedation Scale and the Riker SedationAgitation Scale in evaluating patients' eligibility for delirium assessment in the ICU. Chest. 2012;142(1):48-54.

3. Lin SM, Liu CY, Wang CH, et al. The impact of delirium on the survival of mechanically ventilated patients. Crit Care Med. 2004;32(11): 2254-2259.

4. Pisani MA, Kong SY, Kasl SV, Murphy TE, Araujo KL, Van Ness PH. Days of delirium are associated with 1-year mortality in an older intensive care unit population. Am J Respir Crit Care Med. 2009;180(11): 1092-1097.

5. Khan BA, Zawahiri M, Campbell NL, Boustani MA. Biomarkers for delirium - a review. Journal of the American Geriatrics Society. Nov 2011;59 Suppl 2:S256-S261.

6. Pinto SS, Gottfried C, Mendez A, et al. Immunocontent and secretion of $\mathrm{S} 100 \mathrm{~B}$ in astrocyte cultures from different brain regions in relation to morphology. FEBS letters. 2000;486(3):203-207.
7. Porter JT, McCarthy KD. Astrocytic neurotransmitter receptors in situ and in vivo. Prog Neurobiol. 1997;51(4):439-455.

8. Cerejeira J, Firmino H, Vaz-Serra A, Mukaetova-Ladinska EB. The neuroinflammatory hypothesis of delirium. Acta Neuropathol. 2010;119(6):737-754.

9. Prat A, Biernacki K, Wosik K, Antel JP. Glial cell influence on the human blood-brain barrier. Glia. 2001;36(2):145-155.

10. Koehler RC, Gebremedhin D, Harder DR. Role of astrocytes in cerebrovascular regulation. J Appl Physiol. 2006;100(1):307-317.

11. Han HS, Suk K. The function and integrity of the neurovascular unit rests upon the integration of the vascular and inflammatory cell systems. Curr Neurovasc Res. 2005;2(5):409-423.

12. Kanner AA, Marchi N, Fazio V, et al. Serum S100beta: a noninvasive marker of blood-brain barrier function and brain lesions. Cancer. 2003;97(11):2806-2813.

13. Kapural M, Krizanac-Bengez L, Barnett G, et al. Serum S-100beta as a possible marker of blood-brain barrier disruption. Brain Res. 2002;940(1-2):102-104.

14. Marchi N, Rasmussen P, Kapural M, et al. Peripheral markers of brain damage and blood-brain barrier dysfunction. Restor Neurol Neurosci. 2003;21(3-4):109-121.

15. van Munster BC, Bisschop PH, Zwinderman AH, et al. Cortisol, interleukins and $\mathrm{S} 100 \mathrm{~B}$ in delirium in the elderly. Brain Cogn. 2010;74(1):18-23.

16. Pfister D, Siegemund M, Dell-Kuster S, et al. Cerebral perfusion in sepsis-associated delirium. Crit Care. 2008;12(3):R63.

17. Rasmussen LS, Christiansen M, Rasmussen H, Kristensen PA, Moller JT. Do blood concentrations of neurone specific enolase and S-100beta protein reflect cognitive dysfunction after abdominal surgery?ISPOCD Group. Br J Anaesth. 2000;84(2):242-244.

18. van Munster BC, Korevaar JC, Korse CM, Bonfrer JM, Zwinderman AH, de Rooij SE. Serum S100B in elderly patients with and without delirium. Int J Geriatr Psychiatry. 2010;25(3):234-239.

19. van Munster BC, Korse CM, de Rooij SE, Bonfrer JM, Zwinderman AH, Korevaar JC. Markers of cerebral damage during delirium in elderly patients with hip fracture. BMC Neurol. 2009;9:21.

20. Siami S, Annane D, Sharshar T. The encephalopathy in sepsis. Crti Care Clin. 2008;24(1):67-82, viii.

21. Shimaoka M, Park EJ. Advances in understanding sepsis. Eur $J$ Anaesthesiol Suppl. 2008;42:146-153.

22. Ely EW, Siegel MD, Inouye SK. Delirium in the intensive care unit: an under-recognized syndrome of organ dysfunction. Semin Respir Crit Care Med. 2001;22(2):115-126.

23. Regenstrief Institute, IU Center for Aging Research. Pharmacological Management of Delirium (PMD). Available from: http://clinicaltrials. gov/show/NCT00842608. NLM identifier: NCT00842608. Accessed October 31, 2013.

24. Campbell NL, Khan BA, Farber M, et al. Improving delirium care in the intensive care unit: the design of a pragmatic study. Trials. 2011;12:139.

25. Ely EW, Inouye SK, Bernard GR, et al. Delirium in mechanically ventilated patients: validity and reliability of the confusion assessment method for the intensive care unit (CAM-ICU). JAMA. 2001;286(21): 2703-2710.

26. Sessler CN, Gosnell MS, Grap MJ, et al. The Richmond AgitationSedation Scale: validity and reliability in adult intensive care unit patients. Am J Respir Crit Care Med. 2002;166(10):1338-1344.

27. Wiesmann M, Missler U, Gottmann D, Gehring S. Plasma S-100b protein concentration in healthy adults is age- and sex-independent. Clin Chem. 1998;44(5):1056-1058.

28. Charlson ME, Sax FL, MacKenzie CR, Fields SD, Braham RL, Douglas RG. Assessing illness severity: does clinical judgment work? J Chronic Dis. 1986;39(6):439-452.

29. Jorm AF. The Informant Questionnaire on cognitive decline in the elderly (IQCODE): a review. Int Psychogeriatr. 2004;16(3):275-293.

30. Knaus WA, Draper EA, Wagner DP, Zimmerman JE. APACHE II: a severity of disease classification system. Crit Care Med. 1985;13(10): 818-829. 
31. Routsi C, Stamataki E, Nanas S, et al. Increased levels of serum S100B protein in critically ill patients without brain injury. Shock. 2006;26(1):20-24.

32. Snyder-Ramos SA, Gruhlke T, Bauer H, et al. Cerebral and extracerebral release of protein S100B in cardiac surgical patients. Anaesthesia. 2004;59(4):344-349.

33. Anderson RE, Hansson LO, Nilsson O, Dijlai-Merzoug R, Settergren G. High serum S100B levels for trauma patients without head injuries. Neurosurgery. 2001;48(6):1255-1258; discussion 1258-1260.

34. Scaccianoce S, Del Bianco P, Pannitteri G, Passarelli F. Relationship between stress and circulating levels of S100B protein. Brain Res. 2004;1004(1-2):208-211.
35. Nguyen DN, Spapen H, Su F, et al. Elevated serum levels of S-100beta protein and neuron-specific enolase are associated with brain injury in patients with severe sepsis and septic shock. Crit Care Med. 2006;34(7): 1967-1974.

36. Grandi C, Tomasi CD, Fernandes K, et al. Brain-derived neurotrophic factor and neuron-specific enolase, but not S100beta, levels are associated to the occurrence of delirium in intensive care unit patients. J Crit Care. 2011;26(2):133-137.

37. Kyzas PA, Denaxa-Kyza D, Ioannidis JP. Quality of reporting of cancer prognostic marker studies: association with reported prognostic effect. J Natl Cancer Inst. 2007;99(3):236-243.

\section{Publish your work in this journal}

The International Journal of General Medicine is an international, peer-reviewed open-access journal that focuses on general and internal medicine, pathogenesis, epidemiology, diagnosis, monitoring and treatment protocols. The journal is characterized by the rapid reporting of reviews, original research and clinical studies across all disease areas.
Dovepress

A key focus is the elucidation of disease processes and management protocols resulting in improved outcomes for the patient.The manuscript management system is completely online and includes a very quick and fair peer-review system. Visit http://www.dovepress.com/ testimonials.php to read real quotes from published authors.

Submit your manuscript here: http://www.dovepress.com/international-journal-of-general-medicine-journal 